\title{
Der fiktive Charakter des Ökoinlandsproduktes
}

\section{Ist es möglich, durch Einbeziehung der bisher nicht berücksichtigten Umweltkosten einen Indikator für eine ökologisch nachhaltige Produktion zu entwickeln? Diese Frage war das Thema eines Forschungsprojektes zür Berechnung eines Ökoinland- produktes, das im Auftrag der Europäischen Union u.a. unter Beteiligung des IÖW durchgeführt wurde. Die Autoren plädieren in ihrem Endbericht dafür, als Grundlage für die Berechnung eines derartigen Indikators Umweltnormen zu setzen.}

$\mathrm{E}$ Von Christian Leipert toinlandprodukt (BIP) unter ökologischen Gesichtspunkten unzureichend ist. Die Berechnung des BIP orientiert sich an Marktwerten. Einzig relevante Ausnahme ist die Einbeziehung der unentgeltlich abgegebenen staatlichen Leistungen, die zu Herstellungskosten bewertet werden. Außen vor bleiben die umweltspezifischen Kosten von Produktion und Konsumtion insoweit, als öffentliche Umweltgüter, die kostenlos genutzt werden können, betroffen sind. Dagegen werden die umweltbezogenen Defensivausgaben, mit denen Umweltbelastungen verhindert und/oder reduziert und korrigiert werden, z.T. positiv im BIP verbucht. Kritisch wurde hier in der Debatte um die Aussagefähigkeit des BIP als Wohlfahrtsindikator angemerkt, daß im heutigen System der Volkswirtschaftlichen Gesamtrechnung (VGR) die Umweltkosten des Wirtschaftens unterschlagen werden, dagegen die Maßnahmen zum Umweltschutz und zur Umweltsanierung und -reparatur wachstumssteigernden Charakter haben.

Aufgabe des von der EU finanzierten und im August 1996 abgeschlossenen 20-Monats-Projektes war es (1),

1) theoretisch, methodisch und empirisch gangbare Wege für eine Einbeziehung der Umweltkosten in ein erweitertes ökonomisch-ökologisches Konzept des BIP/Volkseinkommens aufzuzeigen,

2) den Vermeidungskostenansatz zur monetären Bewertung der bisher nicht berücksichtigten Umweltkosten des Wirtschaftens konkret empirisch zu erproben und

3) das Konzept der umweltbezogenen Defensivausgaben theoretisch abzuklären, dessen Rolle im aktuellen Konzept des BIP zu identifizieren und dessen Stellenwert in einem Konzept eines Ökoinlandsproduktes (ÖIP) aufzuzeigen.

In Übereinstimmung mit der Definition im kürzlich revidierten System der VGR (United Nations 1993a) verstehen wir das BIP als Indikator der volkswirtschaftlichen Produktion. Es ist kein Wohlfahrtsmaß. Die wirtschaftliche Wohlfahrt der Bevölkerung hängt von mehr Dimensionen $a b$, als von einem aggregierten monetären Indikator erfaßt werden können. Ziel ist ein Indikator der ökologisch nachhaltigen Produktion durch Einbeziehung der bisher nicht berïcksichtigten Umweltkosten. Dies bedeutet nichts anderes als die Anwendung des „Sustainability“Kriteriums der wirtschaftlichen Entwicklung auf die VGR.

\section{Bewerfung mittels der Vermeidungskostenmethode}

Obwohl dies in vielen Arbeiten zur Entwicklung einer umweltkorrigierten VGR empfohlen worden ist, sind Versuche, monetäre Werte für Umweltschäden zu finden, die eine lange Zeitdimension haben und deren Einschätzung ebenso biophysische Wissensprobleme wie ethische Fragen aufwirft, oft sehr willkürlich. Unserer Auffassung nach kann eine monetäre Bewertung der wirtschaftsbedingten Umweltkosten auf der für die VGR erforderlichen Makroebene nur auf indirektem Wege erfolgen. Wir plädieren daher in unserem Endbericht für ein indirektes und zweistufiges Bewertungsverfahren, das in der Literatur vor allem mit dem Namen von R. Hueting (1980 und 1995) verbunden wird.

Danach müssen zunächst Umweltstandards definiert werden, die die umweltspezifischen Ziele einer Gesellschaft für die Sicherung einer ökologischen Wohlfahrtsbasis zum Ausdruck bringen. In einem zweiten Schritt sind dann die Kosten zu bestimmen, die zur Vermeidung der Umweltbelastungen, die die Umweltstandards verletzen, hätten aufgewendet werden müssen (potentielle oder hypothetische Vermeidungskosten).

Das Statistische Bundesamt hat im Rahmen des Projekts erstmalig derartige (potentielle) Vermeidungskosten geschätzt und die erzielten Ergebnisse zur Konstruktion einer Vermeidungskostenkurve genutzt. Diese Berechnungen wurden für verschiedene Stickstoffverbindungen durchgefuihrt. Die Ergebnisse beziehen sich auf das Vermeidungspotential der wichtigsten Luftschadstoffe Stickoxide $\left(\mathrm{NO}_{\mathrm{x}}\right)$ und Distickoxid $\left(\mathrm{N}_{2} \mathrm{O}\right)$ und für Stickstoffemissionen von öffentlichen Kläranlagen, die in Oberflächengewässer eingeleitet werden.

Die in die Vermeidungskostenkurve einbezogenen Maßnahmen bestehen meist aus End-of-thePipe-Maßnahmen. Für prozeßintegrierte Technologien waren die erforderlichen Daten überwiegend nicht verfügbar. Bei diesen empirischen Pionierarbeiten des Statistischen Bundesamtes zeigte sich drastisch, daß Fortschritte auf dem Wege zu einer Ökologisierung der VGR nicht nur eine Frage von ökonomischer Theorie und geeigneten Konzepten der Rechnungslegung sind, sondern auch eine Frage der Datenverfügbarkeit und ihrer Kompatibilität.

Input-Output-Modelle erleichtern die explizite Repräsentation von technologischen Veränderungen und ihrer Rückwirkungen auf Beschäftigung, Preise, etc. Ein derartiges ökologisch erweitertes Input-Output-Modell enthält Koeffizienten für branchenbezogene Emissionen und für die Inputstruktur der Umweltschutzaktivitäten in den einzelnen Branchen sowie der Umweltschutz- und Sanierungsindustrie.

\section{Kein eindeutiges \\ Ökoinlandsprodukt ermittelbar}

Basierend auf der Idee, kostenminimale Ressourcenallokationsstrategien zur Erreichung von Umweltzielen zu bestimmen, können Opportunitätskosten oder „Trade-Offs“ (in statischer und dynamischer Perspektive) zwischen der Erreichung von Umweltqualitätszielen und dem damit kompatiblen volkswirtschaftlichen Produktionsniveau identifiziert werden. Das ökologisch korrigierte Sozialprodukt ist also hier definiert als höchstmögliches Niveau der Produktion, das unter der Bedingung der Respektierung von unterstellten Umweltstandards modellmäßig ermittelt worden ist. Diese Definition bedeutet zugleich, daß mehr als ein 
Ökoinlandsprodukt (ÖIP) ermittelt werden kann, und zwar in Abhängigkeit von den Umweltstandards, die unterstellt werden.

Die Berechnung eines potentiellen ÖIP entspricht wohlgemerkt nicht der Ermittlung einer tatsächlichen Produktionsgröße. Unter dem Strich ergibt sich also, daß ein eindeutig bewertbares ÖIP nicht ermittelbar ist. Auf indirektem Wege berechnet, hängt der Wert des ÖIP von den Umweltnormen $a b$, die der Berechnung zugrundegelegt werden.

Ein derart modifiziertes System der VGR könnte $\mathrm{zu}$ einem Hilfsmittel für eine empirisch bedeutsame Beurteilung verschiedener Optionen von Umweltpolitik und ihrer Kostenimplikationen werden. Die im Endbericht entwickelten Vorschläge sollen einen Beitrag zu dieser Erweiterung und Rekonstruktion der VGR leisten, die in Reaktion auf neue Politikprioritäten - dem Ruf nach einer ökologisch nachhaltigen Entwicklung gegenüber den Zielen der traditionellen Wachstums- und Fiskalpolitik - notwendig erscheint.

Zur Ermittlung von "Schattenaggregaten“ des BIP, also verschiedenen Varianten eines ÖIP, die mit der Erreichung bestimmter Umweltstandards kompatibel sind, sind verschiedene Ansätze zur Modellierung hypothetischer Strukturen der Wirtschaft denkbar. Eine komparativstatische ex-post Analyse könnte etwa die Auswirkungen der Durchführung (zusätzlicher) technischer Maßnahmen zur Erreichung unterstellter Umweltstandards für eine abgelaufene Periode ermitteln. Eine derartige (kontra-faktische) Modellrechnung, die sich nur auf das vorhandene (umwelt-)technologische Know-how. stützen könnte und von den gegebenen Nachfragebedingungen ausgehen würde, würde erste vorläufige Hinweise auf die erforderliche wirtschaftliche Umstrukturierung als Voraussetzung zur Realisierung der angenommenen Umweltqualitätsziele geben.

Ein mittels einer solchen ex-post Analyse ermitteltes ÖIP wäre vermutlich viel niedriger als im Vergleichsfall einer zukunftsorientierten ex-ante Analyse, in der umweltorientierte technologische Verbesserungen und Nachfragereaktionen aufgrund von Preissteigerungen und Wertwandlungen zugelassen werden. Eine derartige dynamische ex-ante Modellrechnung könnte mit hypothetischen Szenario-Elementen arbeiten und Veränderungen von Konsummustern sowie technische Innovationen zur Erreichung von Umweltstandards berücksichtigen.

\section{- Umweltbezogene \\ Defensivausgaben}

Im Zusammenhang mit der Analyse umweltbezogener Defensivausgaben in einem System einer erweiterten ökonomisch-ökologischen Rechnungslegung mußten mehrere Teilfragen bearbeitet werden:

1) Zunächst war der Bedeutungsgehalt von umweltbezogenen Defensivausgaben zu klären. In welcher Beziehung stehen sie z.B. zu dem gängigen Konzept der Umweltschutzausgaben, deren Ermittlung etwa beim Statistischen Amt der UNO oder auch bei Eurostat, dem Statistischen Amt der EU, im Vordergrund steht?

2) Wie sind die Umweltschutzausgabenrechnung von Eurostat (1994), die gegenwärtig mit großem Elan aufgebaut wird, und die entsprechende Rechnung im SEEA, dem ,System of Integrated Environmental and Economic Accounting“, der UNO (1993b) als mögliche Bausteine für eine Berechnung des ÖIP zu beurteilen?

3) Wie werden Umweltschutzausgaben in der geltenden VGR verbucht und wie wirkt sich die jeweils spezifische Verbuchung der verschiedenen Ausgabenarten auf das nominale und das reale BIP aus?

4) Welche Rolle spielen die umweltspezifischen Defensivausgaben in einem Konzept zur Berechnung eines ÖIP? In welchem Verhältnis stehen die realisierten Umweltschutzausgaben $z u$ den potentiellen Vermeidungskosten, mit denen in unserem Ansatz die bisher nicht erfaßten Umweltkosten von Produktion und Konsum monetarisiert werden sollen?

Die Ergebnisse zu den ersten drei Teilfragen können hier nur in aller Kürze dargestellt werden(2). Die umweltbezogenen Defensivausgaben umfassen vier Teil-Kategorien, und zwar (a) die Ausgaben zur Prävention von Umweltbelastungen, (b) die Restaurierungs- und Sanierungsausgaben, (c) die umweltbezogenen kompensatorischen Ausgaben und (d) die Schadensbeseitigungs- und -behandlungsausgaben. Die Präventions- sowie die Restaurierungs- und Sanierungsausgaben werden i.d.R. als Umweltschutzausgaben zusammengefaßt, so auch von Eurostat im ,European System for the Collection of Economic Data on the Environment" (SERIEE) oder von der UNO im SEEA. Ein Teil der kompensatorischen Ausgaben, die einen eindeutigen und alleinigen Bezug zum Umweltschutz haben, wie die Ausgaben zum passiven Lärmschutz (Lärm- schutzwälle u.ä.), kann noch den Umweltschutzausgaben zugeschlagen werden.

Außen vor bleiben die Schadensbeseitigungsund -behandlungsausgaben. Diese bemühen sich um die Korrektur von Schäden an Menschen, an Gebäuden, an Infrastrukturanlagen, an Werken der Baukunst, etc., die durch Umweltbelastungen hervorgerufen worden sind. Zum Umweltschutz gehören jedoch ausschließlich jene Aktivitäten, mit denen Umweltbelastungen vermieden, reduziert und beseitigt werden.

Umweltbezogene Defensivausgaben bilden den weitestmöglichen Begriff von Ausgaben, die durch negative Umweltwirkungen von wirtschaftlichen Aktivitäten ausgelöst werden. Er deckt sämtliche vier Teil-Kategorien ab. In einer neutralen Formulierung kann man auch von „umweltbezogenen Ausgaben“ sprechen. Der Begriff der (umweltbezogenen) Defensivausgaben ist schon in gewisser Weise belastet. Er verweist auf die Debatte um die Wohlfahrtskritik am BIP, in die er gehört (vgl. dazu ausführlich Leipert 1989). Die Interpretation von „umweltbezogenen Ausgaben“ als Defensivausgaben besagt, daß der Kostencharakter von Umweltschutz- und Umweltsanierungsausgaben betont wird.

\section{Umweltschutzausgaben in einer ökologisch korrigierten Sozialproduktrechnung}

Grundlage einer ökologischen Korrektur des Sozialprodukts ist eine ,erweiterte Umwelt- und volkswirtschaftliche Kostenrechnung“", wie sie im vom Statistischen Amt der UNO herausgegebenen Satelliten-Handbuch zum SEEA entwickelt worden ist. Wenn wir die bisher nicht-berücksichtigten Umweltkosten der Wirtschaft in die VGR integrieren wollen, müssen diese bewertet und monetarisiert werden. Wie oben schon erwähnt, erscheint uns die Ermittlung der Kosten zur Erreichung vorgegebener Umweltstandards als die erfolgversprechendste Methode.

Dabei ist zu beachten, daß die Wahl des Umweltstandards das Ausmaß der ermittelten Umweltkosten des Wirtschaftens determiniert. Im SEEA werden jene Umweltkosten kalkuliert, die durch die inländischen wirtschaftlichen Aktivitäten in der laufenden Periode hervorgerufen werden. Als Umweltstandard wird die von der Vorperiode übernommene und damit zu Beginn der laufenden Periode herrschende Umweltqualität gewählt. Der periodenbezogene Umweltstan- 
dard berücksichtigt also nicht Umweltbelastungen, die durch wirtschaftliche Aktivitäten in Vorperioden verursacht worden sind.

Sicherlich sind auch andere Umweltstandards denkbar. Naheliegend ist vor allem die Orientierung am Maßstab der ökologischen Nachhaltigkeit der Wirtschaftstätigkeit. So orientieren sich Hueting und seine Forschungsgruppe von Statistics Netherlands in ihrem Projekt „Sustainable National Income" für die Berechnung der wirtschaftsbedingten Umweltkosten an Standards einer ökologisch nachhaltigen Nutzung der Naturressourcen (Hueting et al. 1992 und Hueting 1995).

Der Vorteil des SEEA-Standards ist seine Kompatibilität mit der Periodeneinteilung der volksund betriebswirtschaftlichen Rechnungslegung. Die Wahl einer periodenbezogenen Umweltnorm kann jedoch auch schwerwiegende Nachteile mit sich bringen. So wird der Periodenstandard dann, wenn sich die Umweltqualität (oder zumindest einige wichtige Dimensionen) von Periode zu Periode verschlechtert, sukzessive eine immer niedrigere Umweltqualität widerspiegeln. In diesem Fall könnte die Periodennorm „Keep Capital Intact“ völlig falsch interpretiert werden. Die positive Konnotation, die sich mit dieser Regel verbindet, würde die faktische Absenkung der Umweltstandards über mehrere Perioden hinweg faktisch verdecken.

Auch wenn es gelingt, die zu Beginn einer Periode herrschende Umweltqualität über mehrere Perioden $z u$ erhalten, garantiert dies noch nicht eine nachhaltige Sicherung der noch vorhandenen Umweltressourcen. Angesichts einer langen Tradition der Übernutzung der Natur repräsentiert das zu Beginn einer Periode gerade erreichte Niveau der Umweltqualität gegenwärtig nicht notwendig die Bedingungen einer ökologischen Nachhaltigkeit des Wirtschaftens.

\section{- Komplementarität von aktuellen und unterstellten Umweltschutzmaßnahmen}

Präventive Maßnahmen zielen auf die Vermeidung der Entstehung von Schadstoffströmen in der laufenden Periode. Maßnahmen zur Wiederherstellung der Umwelt zielen dagegen auf die Neutralisierung von Umweltschäden, die Sanierung von Flächen und Gewässern, die in der Vergangenheit über eine längere Zeit durch Schadstoffakkumulationen geschädigt worden sind, oder die Renaturierung von zerstörten Landschaften und geschädigten Ökosystemen.
Aktuelle, schon aufgewendete Umweltschutzkosten und potentielle Vermeidungskosten zur Erreichung von (Perioden- oder Nachhaltigkeits-)Umweltstandards stehen in einer komplementären Beziehung zueinander. Dies läßt sich an der Abbildung 1 leicht zeigen. Der Punkt C entspricht einem Niveau der Umweltqualität, das eingetreten wäre, wenn die laufenden Präventionsmaßnahmen nicht durchgeführt worden wären. Punkt $\mathrm{B}$ repräsentiert die aktuelle Umweltqualität, die unter dem Einfluß der aktuell getätigten präventiven Umweltschutzmaßnahmen zustandekommt. Punkt A entspricht dem Umweltqualitätsniveau, das aus der Vorperiode übernommen wurde und angestrebter Umweltstandard in der laufenden Periode ist, der durch die aktuellen und potentiellen präventiven Umweltschutzmaßnahmen erreicht wird.

Die Berechnung von aktuellen und potentiellen Wiederherstellungskosten ist ein Weg, um die Kosten von Umweltschäden $\mathrm{zu}$ ermitteln, die durch nicht-vermiedene Umweltbelastungen in Vorperioden entstanden sind. Es sind Kosten zur Korrektur negativer Wirkungen nicht-nachhaltiger Entwicklungspfade, die in der Vergangenheit gewählt worden sind. Auch hier ergänzen sich wieder aktuelle und potentielle Ausgaben. Das Ergebnis der in der aktuellen Periode durchgeführten Restaurierungs- und Sanierungsbemühungen markiert Punkt D. Dieser stimmt jedoch noch nicht mit den Umweltbedingungen eines nachhaltig aufrechterhaltbaren Niveaus von Produktion und Konsum überein. Um dieses Qualitätsniveau (Punkt E) zu erreichen, müßten zusätzliche Sanierungs-, Renaturierungs- und Schadstoffreduktionsmaßnahmen ergriffen werden.

Bei der Beurteilung der Frage, welche Rolle die verschiedenen Komponenten der umweltbezogenen Ausgaben in einem ÖIP spielen sollen, haben wir uns an dem verursacherorientierten ÖIP-Konzept des SEEA, das im Satellitensystem des neuen SNA eindeutig im Vordergrund steht, orientiert (United Nations 1993b und Stahmer 1995).

Dabei sollen die Umweltkosten der Wirtschaftstätigkeit in der laufenden Periode bestimmt werden, unabhängig davon, ob die nicht-verhinderten Umweltbelastungen im Inland oder im Ausland zu Umweltschäden führen. Die Orientierung an Umweltstandards impliziert u.a., daß keine unbegrenzte Substitution zwischen verschiedenen Kapitalformen (,weak sustainability"), also zwischen reproduzierbarem Produktivvermögen und (teilweise nicht reproduzierbarem) Naturvermögen, zulässig ist. Zugrunde liegt hier das Konzept der „strong sustainability“, wonach Substitutionen nicht im Bereich des „kritischen Kapitals“ zulässig sind. Dies betrifft vor allem die Umweltfunktionen, die Leben ermöglichen und erhalten, - Umweltfunktionen, die nicht durch menschliche Artefakte und technische oder soziale Innovationen ersetzt werden können.

Das Kriterium einer expliziten Berücksichtigung von Umweltschutzausgaben im „cost caused“Konzept eines ÖIP ist, ob sie direkt Niveau oder Qualität des Naturvermögens beeinflussen.

\section{Abbildung 1: Umweltkosten in Abhängigkeit vom Umweltstandard}

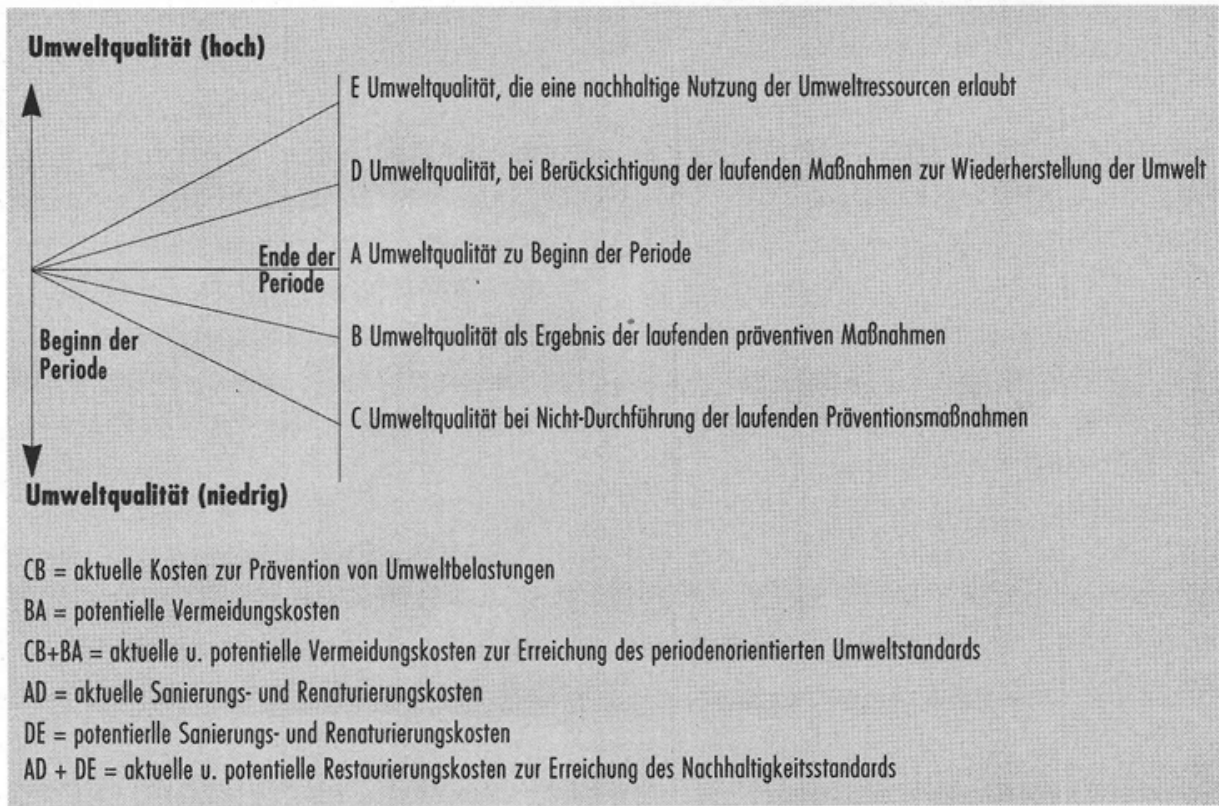




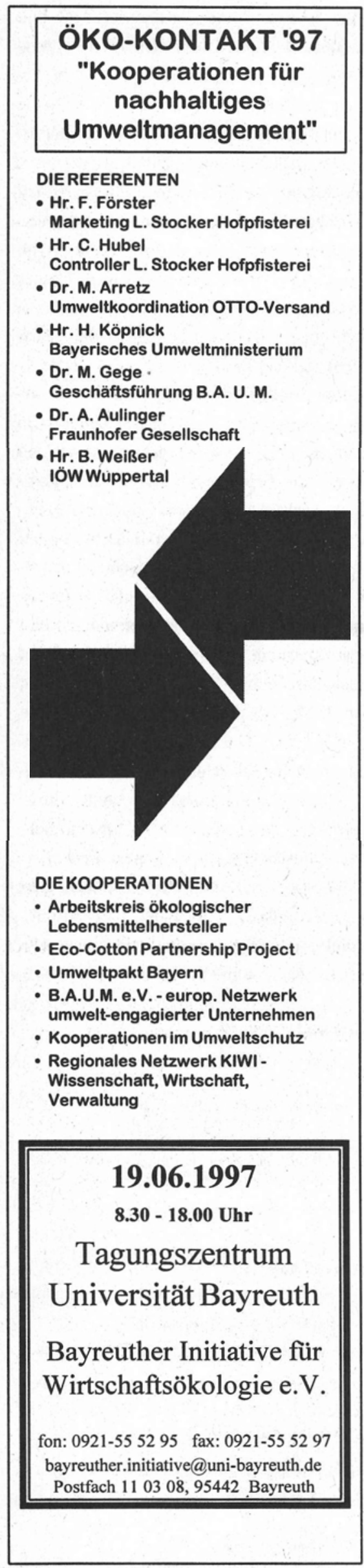

Dieses Kriterium erfillen nur die Restaurierungsausgaben, dagegen nicht die präventiven Ausgaben, die nur zur Erhaltung des Umweltqualitätsniveaus nötig sind, das zu Beginn der Periode geherrscht hat (3). Umweltrestaurierungsausgaben verbessern dagegen das Niveau des Naturvermögens über die Qualitätslinie hinaus, die vom Periodenstandard gefordert wird. Sie sind in diesem periodenorientierten Ansatz substantiell Investitionsmaßnahmen, die zu einem Anstieg bzw. zur Verbesserung des Naturvermögens führen. Diese Investitionen in das Naturkapital bilden einen Korrekturposten, der die Gesamtkosten der Umweltdegradierung und entsprechend den Wert der „Abschreibungen“ auf das Naturkapital vermindert.

Umweltschutzmaßnahmen, die einer Verminderung des Naturvermögens vorbeugen, sind keine echten (Ersatz-)Investitionen. Unter Ersatzinvestitionen versteht man nämlich Maßnahmen zum Ersatz von Produktionsanlagen, die abgenutzt und nach einer gewissen Zeit ausgemustert worden sind. Das ÖIP ergibt sich dann aus folgenden umweltbezogenen Korrekturen des Nettoinlandsproduktes (NIP)

Bruttoinlandsprodukt
- Abschreibungen auf das reproduzierbare
Produktivvermögen
= Nettoinlandsprodukt
+ Bruttoinvestitionen in das Naturvermögen
(Wiederherstellungsausgaben)
- „Abschreibungen“ auf die Abnutzung des
Naturvermögens (potentielle Vermeidungskosten)
$=$ Ökoinlandsprodukt

Soweit die Ausgaben zur Umweltsanierung/renaturierung bisher als privater oder staatlicher Verbrauch verbucht worden sind, hat die Umklassifizierung dieser Ausgaben zu Investitionen in das Naturkapital keine Wirkung auf das ÖIP-Aggregat. Dies wäre anders bei entsprechenden Ausgaben im Unternehmenssektor. Bei einer Umklassifizierung von Vorleistungen in Investitionsausgaben würde diese definitorische Änderung zu einem Anstieg des ÖIP führen.

Die übrigen Kategorien umweltbezogener Ausgaben spielen keine Rolle bei der Kalkulierung des perioden- und verursacherbezogenen Konzepts eines ÖIP. Schadensbehandlungsausgaben beeinflussen nicht das Naturvermögen, sondern behandeln, reparieren und korrigieren Schäden, die Menschen oder menschlichen Artefakten wie Bauten, Kulturdenkmälern und Infra- strukturanlagen zugefügt worden sind. Das Gleiche gilt für den Großteil der kompensatorischen Maßnahmen. Sie korrigieren nur den Verlust von Umweltfunktionen durch die Schaffung eigens produzierter Vorrichtungen, aber nicht den Verlust des Umweltgutes selbst.

\section{Literafur}

Eurostat (1994), SERIEE 1994 Version, Luxembourg. Hueting, R. (1980), New Scarcity and Economic Growth. More Welfare Through Less Production?, Amsterdam: NorthHolland Publ. Company.

Hueting, R. (1995), Schätzung des nachhaltigen Volkseinkommens, in: W. van Dieren (Hrsg.), Mit der Natur Rechnen. Der neue Club-of-Rome-Bericht: Vom Bruttosozialprodukt zum Ökosozialprodukt, Basel-Boston-Berlin, S. 226-247. Hueting, R., Bosch, P. and de Boer, B. (1992), Methodology for the Calculation of Sustainable National Income, WWF International Publication, Gland.

Leipert, C. (1989), Die heimlichen Kosten des Fortschritts. Wie Umweltzerstörung das Wirtschaftswachstum fördert, Frankfurt/M.: S. Fischer Verlag.

Leipert, C. (1995), Defensivausgaben, in: W. van Dieren (Hrsg.), Mit der Natur Rechnen, a.a.0., S. 197-209. Stahmer, C. (1995), Umweltberichterstattung und das System der Volkswirtschaftlichen Gesamtrechnungen, in: W. van Dieren (Hrsg.), Mit der Natur Rechnen, 0.0.0., S. 247-272.

United Nations (1993a), System of National Accounts 1993, New York.

United Nations (1993b), Integrated Environmental and Economic Accounting (SEEA), Handbook of National Accounting, Studies in Methods, Series F, No. 61, New York.

\section{Anmerkungen}

(1) siehe hierzu auch den Artikel „Auf dem Wege zum Ökosozialprodukt?" im IÖW/VÖW-Informationsdienst, H.2, 1995, S. 17-20.

(2) Wer sich für mehr interessiert, sei ouf den Endbericht verwiesen: Brouwer/o'Connor/Leipert/Rademacher/Riege-Wcislo. Methodological Problems in the Calculation of Environmentally Adjusted National Income Figures, Study for the European Commission, December 1996; Introduction \& Part One: Theoretical Perspectives; Part Two: Conceptual and Empirical lssues in Cost-Effectiveness Analysis; Part Three: The Role of Environmental Protection Expenditures in Integrated Environmental and Economic Accounting

(3) Im übrigen sind die Abschreibungen auf den Umweltschutzkapitalstock ebenso wie die Umweltschufzvorleistungen der Marktproduzenten schon bei der Ermittlung des NIP berücksichtigt.

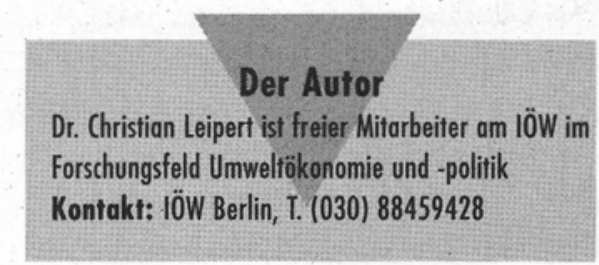


(c) 20I0 Authors; licensee IÖW and oekom verlag. This is an article distributed under the terms of the Creative Commons Attribution Non-Commercial No Derivates License (http://creativecommons.org/licenses/by-nc-nd/3.o/), which permits unrestricted use, distribution, and reproduction in any medium, provided the original work is properly cited. 